\title{
MULTISENSOR DATA FUSION USING MULTIRESOLUSION ANALYSIS (MRA)
}

\author{
Jong-Hyun Park, Ryutaro Tateishi and Ketut Wikantika \\ CEReS(Center for Environmental Remote Sensing) \\ Chiba University \\ pjh@ceres.cr.chiba-u.ac.jp, tateishi@ceres.cr.chiba-u.ac.jp, ketut@ceres.cr.chiba-u.ac.jp
}

KEY WORDS: multisensor, multiresolution analysis(MRA), wavelet transform

\begin{abstract}
The objective of this study is to propose multiresolution data fusion scheme using multiresolution analysis (MRA). Three techniques compared, respectively based on Haar wavelet basis (HWB), Daubechies wavelet basis (DWB), and IHS transform. Daubechies Wavelet Basis (DWB) is far more efficient than the HWB and IHS transform, thus establishing the advantages for data fusion of formally multiresolution analysis. DWB is best image sharpening and maintain the information of original data. Especially, Daubechies wavelet basis is able to enhance image sharpenin $\mathrm{g}$ and preserve spectral information. It has showed the potential application of wavelet transform for higher efficiency for merging spatial and spectral characteristics of multiresolution data.
\end{abstract}

\section{INTRODUCTION}

The great interest in wavelets today is only partly due to their ability to efficiently represent functions with localized features. The interest is also due to the fact that it was discovered that some wavelets might be implemented in an extremely computationally efficient manner by means of what is called a multiresolution analysis (MRA)(Mallat, 1989_a). Just as Fast Fourier Transform algorithms made the Fourier Transform a practical tool for spectral analysis, the multiresolution analysis has made the Discrete Wavelet Transform (DWT) a viable tool for computational time-scale analysis.

In the earliest multiresolution approaches to signal and image processing, the most popular way was to obtain a coarse resolution signal by sub-sampling a fine resolution signal, after linear smoothing, in order to remove high frequencies. Subtracting from each level an interpolated version of the next coarser level then derived a detail pyramid; the best-known example is the Laplacian pyramid (Burt and Anderson. 1983). From a frequency point of view, the resulting difference signals (known as the detail signals) form signal decomposition in terms of band-pass filtered copies of the original signal (Burt and Adelson. 1985).

Remotely sensed images are such a signal. Furthermore, the wavelet transform leads to the concept of multiresolution analysis, where images are decomposed into structures and then analyzed at successive scale (or spatial resolution). The MRA provides a hierarchical pyramid for interpreting the image in terms of structures.

The objective of this part is to propose multiresolution data fusion scheme using wavelet pyramid. Two techniques compared, respectively based on Haar wavelet basis (HWB), and Daubechies wavelet basis (DWB). First, we proposed an optimal wavelet-based fusion rule for an enhancement of spectral and spatial information. Secondly, we calculated the different decomposition level of Haar and Daubechies Wavelet basis to the fused images. Finally, evaluation of fused image is based on urban infrastructures interpretation and made visual and spectral analysis.

\section{DATA SOURCES AND PRE-PROCESSING}

Data sources included IRS-1C and Landsat TM data. Landsat TM had acquired on Sep.1, 1996 and IRS-1C panchromatic image acquired on Nov. 6, 1996, respectively. Landsat TM and IRS-1C images are registered and resampled. IRS-1C pan data is $5.8 \mathrm{~m}$, the first commercial satellite imagery, which breaks the $10 \mathrm{~m}$ barrier and offers regular repeat coverage.

Data fusion is dividing into three stages in this study. The first is image registration. We first correspond the images to a map-based reference system using a database of image chips with associated map coordinated. Once this image-to-map correspondence is completed. This correction was applied to the image coordinates of the ground control points (GCP) in order to compute a mapping function which is not affected by terrain induced image distortions. The resampling procedure is applied to cubic convolution. This procedure is based on a complex function that determines the value to be given to new pixel using the weighted response of surrounding pixels and is to be preferred urban scenes (Forster, 1985).

We first correspond the images to a map-based reference system using a database of image chips with associated map coordinated. Once this image-to-map correspondence is completed. The RMS error is obtained within 0.5 pixels using 
ER Mapper (Earth Resource Mapping, 1995). This correction was applied to the image coordinates of the ground control points (GCP) in order to compute a mapping function which is not affected by terrain induced image distortions. The geometric rectification of the Landsat TM and IRS-1C imagery then combines an affine transformation.

\subsection{Data mining}

The basis concept of the data fusion of this research departs from data mining. The data mining is extract effective information out of a lot of data (Glymour et al., 1997). The rule nature and causality that are hiding into a lot of data by data mining can be extracted. In other words, it is to be connected to the stage of data fusion from the concept of data mining.

This stage involves band mining using all the bands of Landsat TM (except band 6). Band mining is using OIF (optimum index factor)(Chavez et al. 1984). It is based on the amount of total variance and correlation within and between various band combinations. The algorithm used to compute the OIF from any subset of three bands is.

$$
\text { OIF } \quad ? \underset{k ? 1}{?} s_{k}^{3} / \underset{k ? 1}{?} \operatorname{Abs} ? r_{j} ?
$$

$s_{k}$ is the standard deviation of band k here and $A b s r_{j}^{\prime \prime}$ " is the absolute value of the correlation coefficient in 3 bands. It assumed to have the smallest redundancy information beside; it has much information, as the value of OIF is a big. It is based on the amount of total variance and correlation within and between various band combinations.

The three-band combination with the largest OIF generally will have the most information with the least amount of duplication. We obtained two sets of band combination- 5/4/3 ? computed from OIF. Landsat TM, OIF of band 3,4,5 is highest, continue with band $1,4,5$ and 4,5,7.

\section{WAVELET TRANSFORM}

The wavelet transform allows multiresolution decomposition, analysis and reconstruction of remotely sensed images. A wave is usually defined as an oscillating function of time or space, such as a sinusoid. Fourier analysis is wave analysis. A wavelet is a small wave, it still has the oscillating wave like characteristic but also has the ability to allow simultaneous time and frequency (Mohanty, 1997).

Wavelet is an active field of signal analysis approximation theory that describes the frequency content of a signal in time and combines powerful new mathematical methods for representing functions (Li et al., 1995). Wavelets are another type of filter family(Strang and Nguyen, 1997). They are constructed from a mother wavelet, which can be any admissible function. A family is constructed by dilating and translating the mother wavelet by different amounts. Prominent examples of wavelet families are the Daubechies, Coiflet, Haar, and Symmlet(Burt and Adelson, 1985). A signal or function $\mathrm{f}(\mathrm{t})$ can be better analyzed if expressed as a linear decomposition by

$$
f(t) ? ?_{j} a_{j} ?_{j}(t)
$$

$a_{j}$ is the real value expansion coefficient and ${ }_{j(t)}$ is called the expansion set.

The Fourier transform maps a one-dimensional function of continuous variable into a one-dimensional sequence of coefficient. For the wavelet transform, a two-parameter system is constructed as

$$
f(t) ? ?_{k}^{?} ?_{j} a_{j, k} ?_{j, k}(t)
$$

$a_{j, k}$ are called the discrete wavelet transform(DWT) of $\mathrm{f}(\mathrm{t})$ and ${ }_{j, k}$ called the generating wavelet or mother wavelet(e.g. Haar, Daubechies, Coiflet, etc).

\subsection{Discrete wavelet transform (DWT)}

The discrete wavelet transform (DWT), as developed by Daubechies (1992), has many similarities to the fast Fourier transform (FFT). The transform for the FFT yields a unique decomposition in terms of continuous sine and cosine as the transform output. The wavelet transform yields a decomposition that is neither continuous nor unique (Fig. 1). The primary advantage of the DWT is that it does not have the limited time-frequency resolution of the FFT and thus provides more accurate representation of the input (Rioul and Vetterli, 1991). 


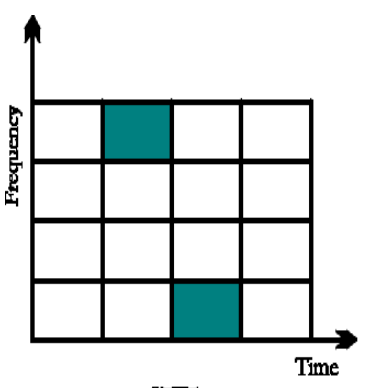

STFT

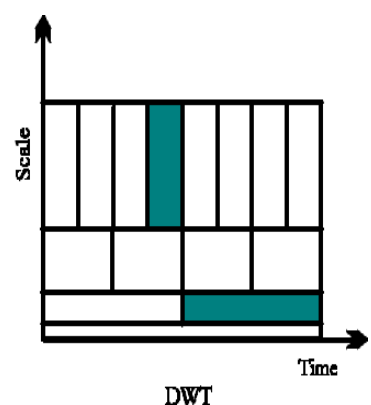

DWT

Figure 1. Discrete wavelet transform

These are three characteristics that are more specific to wavelet expansion. 1) The wavelet systems are generated from a single scaling function or wavelet by simple scaling and translation. The two-dimensional parameterization is achieved from the function called mother wavelet.

$$
\begin{gathered}
f(t)=\sum_{j, k} a_{j, k^{2}} 2^{3 / 2} \psi\left(2^{j} t-k\right) \\
f(t)=\sum_{j, k} a_{j, k} \psi_{j, k}(t)
\end{gathered}
$$

$¥$ : wavelet

$$
\varphi_{k(t)}=\varphi(t-k)
$$

$\mathrm{k}$ : scaling parameter, $\mathrm{j}$ : translation parameter, $¥:$ scale function

$\mathrm{f}(\mathrm{t})$ is the set of all integers and $2^{j / 2}$ maintains a constant norm independent of scale $\mathrm{j}, 2$ ) Almost all useful wavelet systems also satisfy the multiresolution conditions. This means that if a set of signals can be represented by a weighted sum of ? $(2 t ? k$, ) then a larger set of signals can be represenated b y a weighted sum of ? $(t$ ? $k), 3)$ The lower resolution coefficients can be calculated from the higher resolution coefficients by a tree-structured algorithm called filter bank or DWT. Filter bank will separate signal into two frequency band, low and high.

\subsection{Multiresolution Analysis (MRA)}

The MRA is based on the decomposition of the image into multi channel based on the their local frequency content. The wavelet transform provides a framework to decompose images into a number of new images, each multiresolution analysis based on the wavelet theory permits the introduction of the concepts of details one of them with a different degree of resolution. The idea behind image pyramids is to generate a number of homogeneous parameters that represent the response of a bank of filters at different scales and possibly different orientations. There are many different types of filters that can be used for this purpose. Multiresolution approach using wavelets is an efficient and effective way of representing data (Rosenfeld, 1984). According to Mallat (1989_b), a primary advantage of the multiresolution approach using wavelets is its spatial orientation selectivity.

$$
\begin{aligned}
& B(k) ? ?_{m} L(m ? 2 k) A(m) \\
& C(k) ? ?_{m} H(m ? 2 k) A(m)
\end{aligned}
$$

$\mathrm{L}$ and $\mathrm{H}$ is expansion constant of coefficients. Representative coefficients are used Haar or Daubechies wavelet coefficient. A is upper-band input, B and C are output of low and high frequency respectively (Fu et al., 1984). This is required down sampling for decomposition and up sampling for reconstruction.

\section{SCHEME OF MULTISENSOR DATA FUSION USING OPTIMAL WAVELET-BASED FUSION RULE (OWFR)}

The objective of this study is to propose data fusion scheme using wavelet-based multiresolution analysis. Wavelet-based data fusion can be performed in two ways: 1) by replacing some wavelet coefficients of the multispectrd 
data by corresponding coefficients of high resolution data and 2) by adding high resolution coefficients to the multispectral data. Here, we use the discrete wavelet transform algorithm known as Haar and Daubechies for decompose the data into wavelet coefficients.

The method is applied to fuse Landsat TM and IRS-1C data. First, we investigated standard wavelet decomposition and optimal wavelet decomposition for an enhancement of spectral and spatial information. Secondly, We calculated the different decomposition level of Haar and Daubechies Wavelet basis to the fused images. Finally, evaluation of fused image is based on urban objects interpretation and is made by visual and spectral analysis. Wavelet transform is first performed on each source images, and then a fusion decision rule is generated based on a set of fusion rules (Fig. 2). The fused wavelet coefficient can be constructed from the wavelet coefficients of the source images according to the fusion decision rule. Finally the fused image is obtained by performing the inverse wavelet transform.

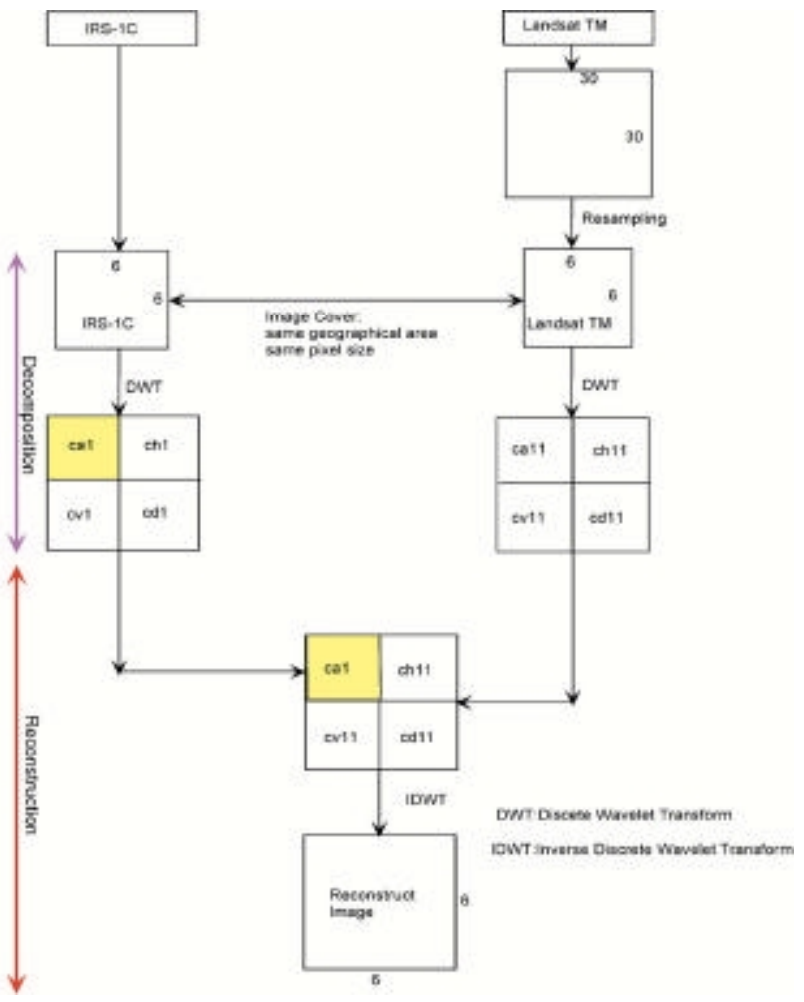

Figure 2. Concept of data fusion using MRA
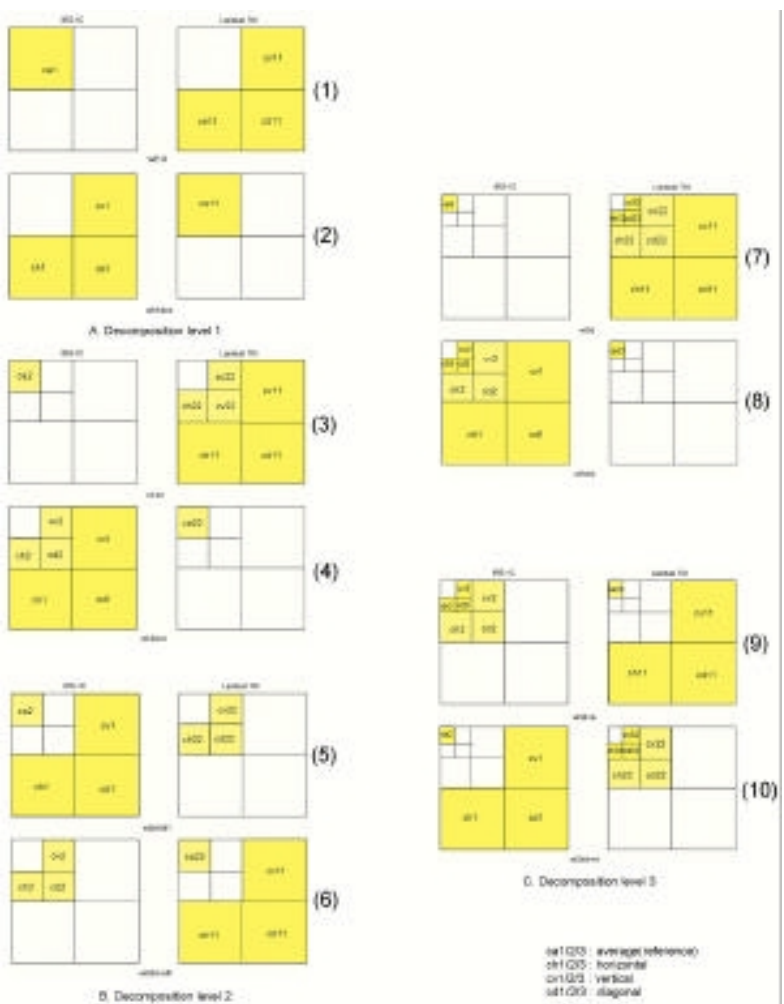

Figure 3. Scheme of a decomposition level for optimal wavelet transform

From the Figure 3, it is show that the fusion rules are playing a very important role during the fusion process.

\section{RESULTS}

This section presents about a quantitative evaluation of optimal wavelet-based fusion rule (OWFR) algorithms performing a pixel-based data fusion from Landsat TM and IRS-1C data having different spatial resolution. The wavelet transform leads to the concept of multiresolution analysis (MRA), where images are decomposed into structures and then analyzed at successive spatial resolution. The MRA provides a hierarchical pyramid for interpreting the image in terms of structures.

A set of quantitative parameters has been recently proposed. Both visual quality, regards as contrast, presence of fine details, and absence of impairments and artifacts, and spectral fidelity as a concerned and embodied in the measurements (Wald, 1997).

\subsection{Visual analysis for urban infrastructures interpretation by fused images}


Roads and highways are the most important regions from traffic and living conditions. Therefore housing development and commercial building can be found along streets. Fused image of Landsat TM and IRS-1C of the new town can be detected 10-30 m width asphalt and concrete pavements. Namely, the main roads and street detected by fused image of high-resolution satellites and Landsat TM (Fig. 4). Highways can be easily extracted as well roads. Roads can be collected easily in new subdivisions and developments, while there is difficulty in core and old urban areas due to the small width of the street, the shadows and the foliage of trees except for the wide roads. Railways can be easily detected but contextual information is required to identify it. Number of tracks was not visible.

Buildings (housing development and commercial building) were found that it along streets. It was difficult to identify buildings and more difficult to extract the shape of the building in both urban and rural areas, except for large complexes. Visually, the pixel value of surfaces among different building roof (residential, industrial, commercial) are very similar to the pixel values of bare soil on the surrounding new and old region of town. The material of roofs is being change in new town, consequently also the characteristics of the remote sensed data change.

The spatial resolution of the IRS-1C imagery is at the upper limit of the range, wh ile the 6bits dynamic range will result in a lower contrast. The resampled gray levels of the image ranged from about 70 to 255 for both the entire scene and the sub-scenes (Armenakis and Savopol, 1998). The 6bits quantization of the IRS-1C results in lower contrast imagery, thus making detection and identification of features more difficult. But, with high-resolution imagery the very small change can be detected on the ground. The fine spatial resolution of the imagery, coupled with the increased temporal resolution and the ease of image registration, will greatly enhance infrastructure detection and monitoring (Park et al., 1999).

The ratio of the spatial resolution of IRS-1C and Landsat TM data becomes 1:5. Therefore, it is thought that it is unable to apply the data fusion method of established in accordance with high resolution data easily unlike, in the case of the fusion of SPOT and Landsat TM of established. Thereupon, there is need to confirm the accuracy of fused image, and determine what kind of change there is by the ratio of the quality and spatial resolution of each original image

\subsection{Statistical analyses}

\section{Spatial quality}

Another representation of assessment between channels is correlation matrix. This is a normalized form of the covariance matrix that has values of 1 along the diagonal with all off-diagonal values being in the range ?1 to 1 .

$$
c_{i j} ? \frac{?_{i ? 1}^{n}\left(x_{i} ? \bar{x}\right)\left(y_{i} ? \bar{y}\right)}{\sqrt{?_{i ? 1}^{n}\left(x_{i} ? \bar{x}\right)^{2} \sqrt{?_{i ? 1}^{n}\left(y_{i} ? \bar{y}\right)^{2}}}}
$$

Most of the evaluation is based on visual examination. In this study we used the correlation coefficients between the original IRS-1C image and merged images as an index of the spatial quality. This measurement is based on the fact that the spatial information unique in IRS -1C panchromatic is mostly concentrated in the high frequency domain. The higher correlation between IRS-1C panchromatic indicates that more spatial information from IRS-1C image is incorporated during fusing. Table 1 shows the correlation coefficients of merged images and IRS-1C panchromatic image. $3 \mathrm{H}$ has best spatial information derived from IRS-1C.

\begin{tabular}{c|cc}
\hline Correlation coefficient & IRS-1C/images & Average of coefficient \\
\hline IRS-1C & 1 & - \\
IHS_R & 0.995 & 0.610 \\
IHS_G & 0.436 & 0.733 \\
IHS_B & 0.401 & \\
Haar_R (no. 9) & 0.772 & 0.737 \\
Haar_G (no.9) & 0.737 & \\
Haar_B (no. 9) & 0.69 & \\
DB_R (no. 9) & 0.776 & 0.744 \\
DB_G(no. 9) & 0.693 & \\
DB_B (no.9) & & \\
\hline
\end{tabular}

Table 1. Correlation matrix between IRS-1C image and fused images 
Table 1 shows the correlations between the results by the algorithms. Note that the correlations coefficients of the DB and Haar (no. 9) are higher than that of IHS. There is strong correlation between the DB (no.9) merged images and spatial information of panchromatic image (IRS-1C). This high correlation produce images that are closer to the IRS -1C image than desirable.

\section{Quantity of information}

The entropy presents information measurement involved in single band. The larger the entropy is the more information of the fused images has accumulated, and DB (no. 9) fusion method presents the best result (Table 2).

The image, the distribution of the density level has inclination, has little quantity of information, because it has redundancy without using the level number that was given effectively. Fused image has also a lot of correlations among adjacent pixels. Each band of the multispectral image is not independent information and there is a correlation fairly among the band. It is said that the effective quantity of information that is included to a satellite image. As a result, it becomes small to about $1 / 10$ of the value that added the bit number of the digital image. Therefore, by removing of redundancy, can be compress the data dimension. This is connected to the extraction of effective data simultaneously.

Table 2 shows that the results of entropy calculation for original and fused images. Note that the entropy of DB (no. 9) are higher than that of IHS, and Haar (no. 9). This high entropy produce fused images that are much more information.

Consequently, DB (no.9) transform, we can adjust easily the extraction of quantity and spatial information from Landsat TM and IRS-1C.

\begin{tabular}{c|cc}
\hline Image & Entropy & \multicolumn{2}{|c}{ Average } \\
\hline IRS-1C & $7.25 \mathrm{E}+10$ & \\
TM_Red & $1.69 \mathrm{E}+10$ & $1.6357 \mathrm{E}+10$ \\
TM_Green & $1.94 \mathrm{E}+10$ & \\
TM_Blue & $1.28 \mathrm{E}+10$ & \\
\hline IHS_R & $8.31 \mathrm{E}+10$ & $3.7283 \mathrm{E}+10$ \\
IHS_G & $9.45 \mathrm{E}+09$ & \\
IHS_B & $7.50 \mathrm{E}+09$ & \\
Haar_R (no. 9) & $3.69 \mathrm{E}+10$ & \\
Haar_G (no. 9) & $4.06 \mathrm{E}+10$ & \\
Haar_B (no. 9) & $3.44 \mathrm{E}+10$ & \\
DB_R (no. 9) & $3.63 \mathrm{E}+10$ & \\
DB_G(no. 9) & $4.01 \mathrm{E}+10$ & \\
DB_B (no. 9) & $3.84 \mathrm{E}+10$ & \\
\hline
\end{tabular}

Table 2. Entropy of original images and fused images

\section{CONCLUSIONS}

Multisensor data fusion is an effective means of exploiting the complementary nature of different data type. This study presents the utility of wavelet transform for data fusion purpose. The work have made a discussion on the effort of different decomposition level of Haar and Daubechies wavelet basis to the fused images and have found that the quality of fused image by the different wavelet basis. In this case, the Haar and Daubechies wavelet basis with decomposition level 3 exhibits the best result. Wavelet transform has showed the potential application for higher accuracy for merging spectral and spatial information of multiresolution remotely sensed data.

By statistical and visual analysis, we can draw the following conclusions. 1) Two decomposition model (Haar and Daubechies) produce a little different results, 2) the optimal wavelet decomposition shows to be far more efficient, and 3) the longer the filter length, the sharper the spatial enhancement (DB10: no. 9 of Fig. 4), the best wavelet is Daubechies wavelet basis with at level 3.

Daubechies Wavelet Basis (DWB) is far more efficient than the Haar Wavelet Basis (HWB), thus establishing the advantages for data fusion of formally multiresolution analysis. DWB is best image sharpening and maintain the information of original data. Especially, Daubechies wavelet basis is able to enhance image sharpening and preserve spectral information. It has showed the potential application of wavelet transform for higher efficiency for merging spatial and spectral characteristics of multiresolution data. But, multisensor data fusion, it is difficult to achieve optimally spectral and spatial results simultaneously because the information from these different sensors may degrade 
each other.

Consequently, fusion rule of Haar and Daubechies level 3 is shown that spatial resolution was enhanced rather than others rule. This result indicates that optimal wavelet -based fusion rule is upper-level decomposition with panchromatic data of level 3. It was used panchromatic data as for average (reference).

\section{REFERENCES}

Armenakis, C. and Savopol, F., 1998, MAPPING POTENTIAL OF THE IRS-1C PAN SATELLITE IMAGERY, IAPRS, Vol. 32, Part 4 i GIS-Between Visions and Applications; , Stuttgart, pp. 23-26.

Burt, P.J. and Anderson, E. H., 1983, The Laplacian pyramid as a compact image code, IEEE Transactions on Communications, vol. 31, pp. 532-540.

Burt, P.J.,and Adelson, E.H., 1985, Merging images through pattern decomposition. Proceedings of SPIE, Vol. 575, pp. 173-181.

Chavez, S.C. P.S.Jr., Guptill, and Bowell, B.A., 1984, Image Processing techniques for Thematic Mapper data, Proceedings, ASPRS-ACSM Technical Papers Vol. 2, pp. 728-742.

Daubechies, I., 1992, Ten lectures on wavelets, SIAM, Philadelphia, Pennsylvania.

Earth Resource Mapping, 1995, ER Mapper 5.0 Applications, Earth Resource Mapping Pty Ltd, pp. 1 -18.

Forster, B.C. 1985, An examination of some problems and solutions in monitoring urban areas from satellite platforms, International Journal of Remote Sensing, 1985, VOL. 6, NO. 1, pp. 139-151.

Fu, K., Huang, T.S. and Schroeder, MR., 1984, Multiresolution Image Processing and Analysis, Springer -Verlag Berlin Heidelberg, Part III, pp. 38-101.

Glymour, C., Madigan, D., Pregibon, D., and Smyth, P., 1997, Statistical Themes and Lessons for Data Mining, Data Mining and Knowledge Discovery, Vol. 1, pp. 1-28.

Rosenfeld, A., 1984, Multiresolution image processing and analysis, Springer-Verlag, Berlin.

Mallat, S.C.,1989_a, A theory for multiresolution signal decomposition: the wavelet representation, IEEE Transactions on pattern Analysis and Machine Intelligence, Vol. 11, pp. 674 -693.

Mallat, S.C., 1989_b, Multifrequency channel decompositions of images and wavelet models, IEEE Tran. Acoustics, Speech and Signal Processing, vol. 37, pp. 2091-2110.

Manjunath, H. Li, B.S. and Mitra, S.K., 1995, Multisensor Image Fusion Using the Wavelet Transform, Graphical Model and Image Processing, Vol. 57, No. 3, pp. 235-245.

Mohanty, K.K., 1997, The wavelet transform for local image enhancement, International Journal of Remote Sensing, Vol. 18, No. 1, pp. 213-219.

Park, J.H. , Tateishi, R., Ketut Wikantika and PARK, J.G., 1999, The potential of High Resolution Remotely Sensed Data for Urban Infrastructure Monitoring, IGARSS i99, Hamburg Germany, BB37_PARJ.

Rioul, O., and Vetterli, M., 1991, Wavelets and signal processing, IEEE Signal Processing Magazine, Vol. 8, pp. 14 -38. Schwengerdt, R.A., 1997, Remote Sensing Models and Methods for Image Processing $2^{\text {nd }}$ Ed., ACADEMIC PRESS, Chap. 4.

Shannon, C., Bell System Tech. J., 1948. Reprinted in Shannon, C. E. and Weaver, W., The Mathematical Theory of Communication, University of Illinois Press, Urbana, 1949.

Strang, G and Nguyen, T., 1997, Wavelets and Filter Banks, Wellesley-Cambridge Press, Chapter 1.

Wald, L., Ramchin, T., and Mangolini, M., 1997, Fusion of Satellite Images of Different Spatial Resolutions: Assessing the Quality of Resulting Images, Photogrammetric Engineering \& Remote Sensing, Vol. 63, No. 6, pp. 691-699.

Zhu, C and Yang, X., 1998, Study of remote sensing image texture analysis and classification using wavelet, International Journal of Remote Sensing, Vol. 19, No. 16, pp. 3197-3203. 

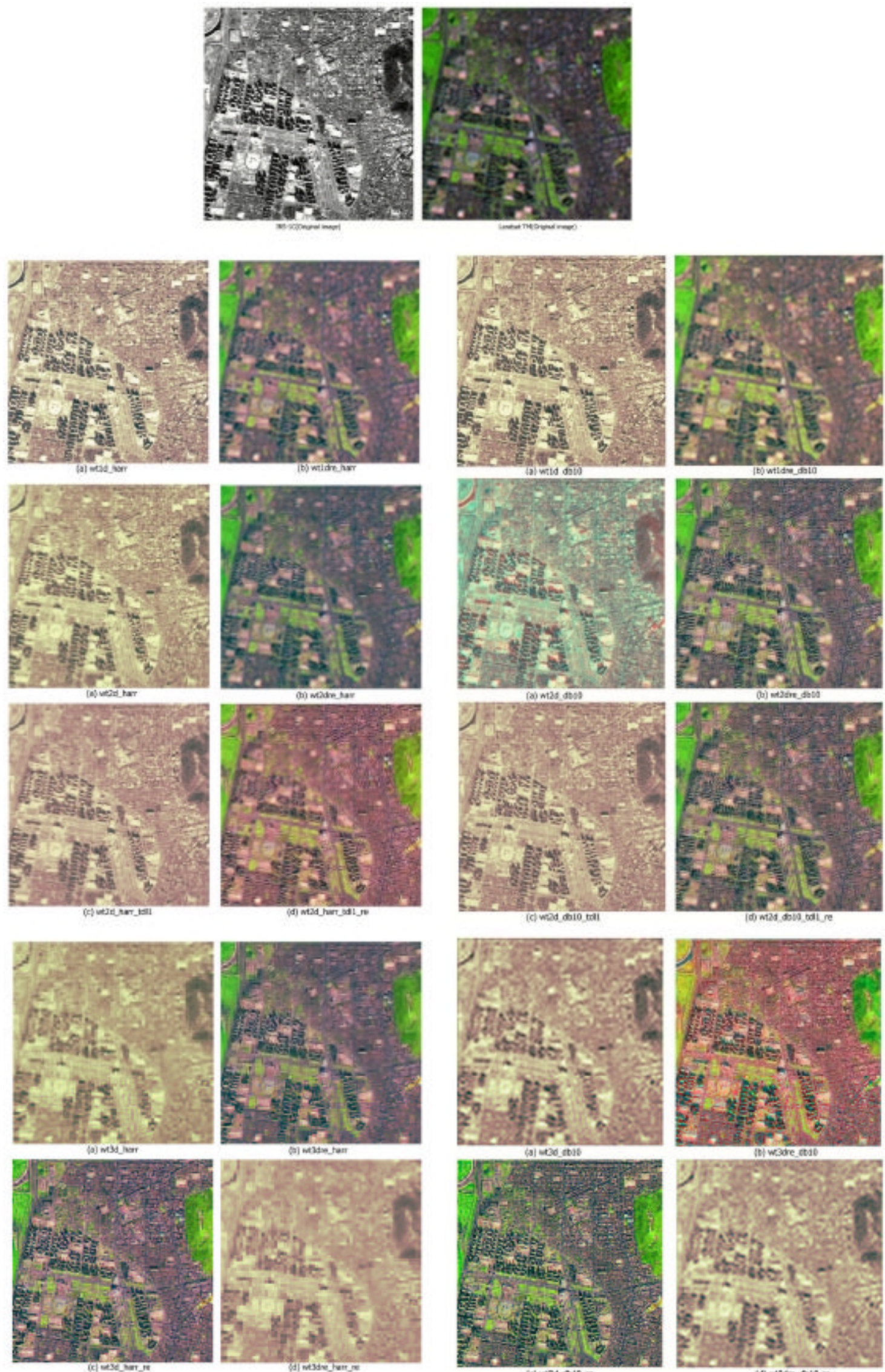

Haar Wavelet Basis
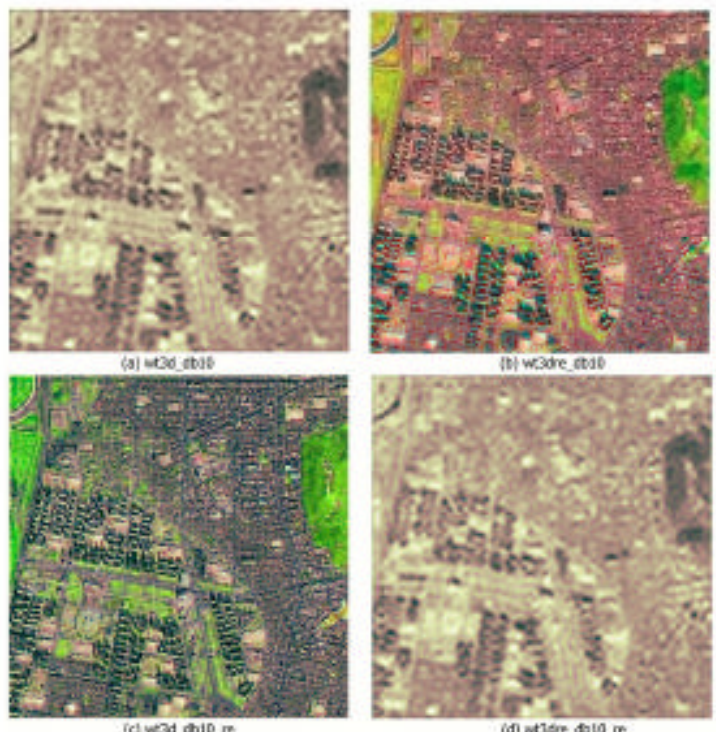

Daubechies Wavelet Basis

Figure 4. Fused images from optimal wavelet-based fusion rule 